\title{
Efficiency Measurement of Compressed Air Compressors Using High Availability SoC With 1002 Redundancy Architecture
}

\author{
MOHAMED ABDELAWWAD ${ }^{\mathrm{a}, 1}$, EIKE HAHN ${ }^{\mathrm{a}}$ and JOSEF BOERCSOEK ${ }^{\mathrm{a}}$ \\ JULIAN FAIRBROTHER $^{\mathrm{b}}$, TAREK AL SHAHADAT ${ }^{\mathrm{b}}$ and PETER OTTO ${ }^{\mathrm{b}}$ \\ ${ }^{\mathrm{a}}$ ICAS, Institute for Computer Architecture and System Programming, University of \\ Kassel, Kassel, Germany \\ ${ }^{\mathrm{b}}$ Postberg + Co. GmbH, Kassel, Germany
}

\begin{abstract}
Compressed air systems are essential components in various industrial and everyday applications. The efficiency of these systems is very important due to their role in the energy consumption of industrial plants. To increase efficiency, a new concept for compressed air compressors based on Industry 4.0 is presented. Due to the aggressive environmental conditions in which the compressed air compressors operate, a new design of a $\mathrm{SoC}$ with high availability based on 1002 redundancy architecture is developed.
\end{abstract}

Keywords. Smart compressed air compressor, compressor efficiency, isentropic efficiency, 1002 redundancy architecture, high availability SoC, volume flow measurement

\section{Introduction}

One of the most important components in many areas of daily life and industry are the Compressed Air Systems (CASs) [1]. Therefore, the market size was estimated at USD 31.26 billion in 2018 and is expected to reach USD 32.73 billion by 2025 [2]. However, the efficiency of the CASs is a major dilemma as 5 to $10 \%$ of electricity consumption in industrial applications is used by them [3,4]. Therefore, measuring and improving efficiency of CASs plays an important role in reducing energy consumption and result in potential economic savings [5].

The current strategic goal of industrial development is to implement the concept of Industry 4.0 [6]. In this concept, intelligent sensor technology is developed to achieve the so-called Cyber Physical Systems (CPS), where a connection between the physical and virtual space is established and improved. To increase reliability and availability, additional functionalities such as fault prediction and self-adaptation are integrated into the new CPSs [7]. For higher level, semantic communication the CPS supports the OPC UA protocol via an ethernet interface.

\footnotetext{
${ }^{1}$ Corresponding Author: Research Engineer, Institute for Computer Architecture and System Programming, University of Kassel, Wilhelmshöher Allee 71, 34121 Kassel, Germany; E-mail: m.abdelawwad@unikassel.de.
} 
Wear occurring in industrial compressed air compressors is a long-ignored subject which can lead to potentially efficiency degradation. For example, the efficiency of compressors according to [8] shows a degradation of up to $45 \%$ over 30 years, which is a typical life cycle for compressors.

The current Life Cycle Cost (LCC) calculation is based on fixed compressor efficiency values given by the manufacturers which cannot be guaranteed over the whole lifecycle of the compressor. Therefore, the continuous efficiency assessment can be used to get a proper LCC calculation. Changes in efficiency can also be used to initialize maintenance measures to avoid increases in energy consumption and emissions.

The paper at hand presents a novel solution for continuous efficiency assessment of compressed air compressors. The possibility to monitor efficiency degradation over time of on-site in operating compressed air compressors could give vital insights into energy consumption reduction potentials and emission reduction in the industrial sector.

Another potential issue with CASs is that, in most cases, compressed air compressors are installed and operated in an environment that is aggressive to electronic components. This can lead to availability problems. Therefore, a new design of compressed air compressor efficiency assessment based on a 1002 system architecture is proposed.

\section{Efficiency calculation of industrial compressor systems}

In the last years, there have been several significant international developments in the field of regulations and energy efficiency standards for air compressors. The European Union released the Ecodesign preparatory study on compressors (ENER Lot 31) [9], the US Department of Energy (DOE) issued the Energy Conservation Standards for Air Compressors [10] and the Peoples Republic of China has released GB 19153-2019 Minimum allowable values of energy efficiency and energy efficiency grades for displacement air compressors [11].

These studies setup frameworks for compressor efficiency, i.e. differentiation between different compressor types and define metrics to access the efficiency and costs of a compressed air compressor. So called minimum energy performance standards (MEPS) are adopted under which manufacturers of compressed air compressors are obliged to reduce emission and the energy consumption throughout the product life cycle.

Manufacturers are required to comply to the new standards and have to test their machines according to the regulations. Compliance with DOE rule is required after January 10, 2025. The Ecodesign regulation is still in process.

For existing in-service compressors an energy audit is an effective instrument to detect optimization potentials. A comprehensive evaluation of the compressed air system should expose over-sized, old and inefficient equipment, equipment without proper control, leakages and inappropriate applications. ISO 11011:2013 Compressed air - Energy efficiency - Assessment recommends a holistic approach and considers the whole compressed air system as well as heat recovery. Correct measuring and the right interpretation of the collected data are identified as the basis for efficient usage and predictive maintenance.

To determine the efficiency and proper operation of a machine the most relevant international performance standards and test methods for air compressors are ISO 1217:2009, Displacement Compressor Acceptance Tests [12], ISO 5389:2005, Turbo 


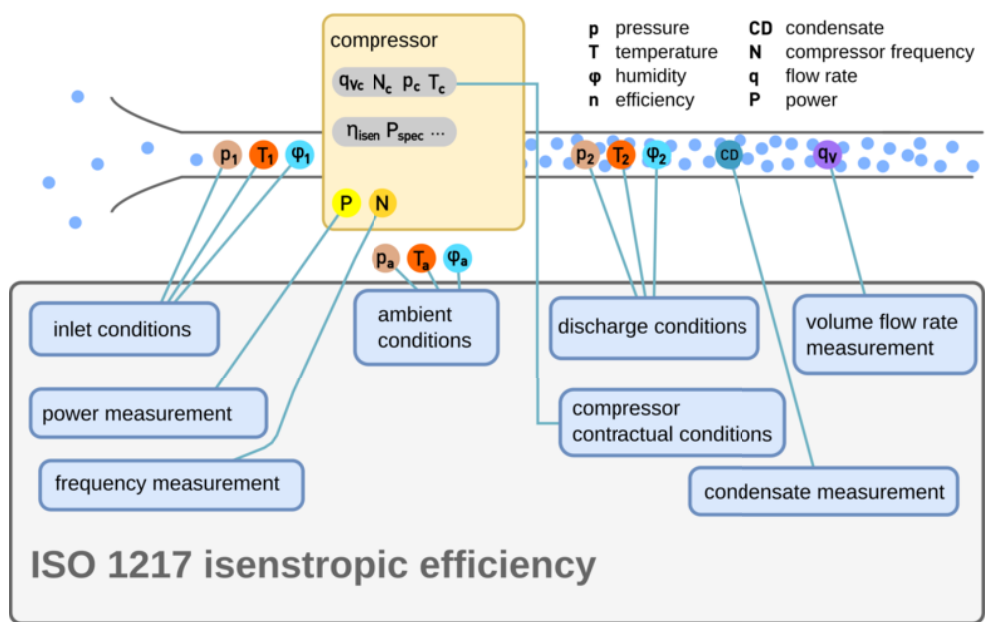

Figure 1. Isentropic efficiency according to ISO 1217 [12].

Compressors - Performance test code [13] and ASME PTC 10-1997, Performance Test Code on Compressors and Exhausters [14].

ISO 1217 specifies operating and testing conditions in arbitrary operating points (full load, part load). Ambient, inlet and discharge conditions, energy consumption, condensate and the volume flow rate have to be measured. Specific test procedures, depending on the type of compressor, should be carried out at certain conditions to assert the compliance to the declared output.

The main metric, adopted by all international regulations, is the isentropic efficiency (figure 1), i.e. the ratio of the theoretical isentropic power to the actual power required for a compression process. The isentropic efficiency makes it possible to easily identify compressor losses compared to an ideal standard and cannot be misunderstood as no conversion of units is required.

$$
\eta_{\text {isen }}=\frac{P_{\text {isen }}}{P_{\text {real }}}
$$

Where:

$\eta_{\text {isen }}$ : isentropic efficiency

$P_{\text {isen }}$ : isentropic power

$P_{\text {real }}$ : electric input power of package

$$
P_{\text {isen }}=q_{V} \cdot \frac{p_{1} \cdot \kappa}{\kappa-1} \cdot\left[\left(\frac{p_{2}}{p_{1}}\right)^{\frac{\kappa-1}{\kappa}}-1\right]
$$

Where:

$q_{V}$ : volume flow rate

$p_{1}$ : inlet pressure 
Table 1. Parameters according to Ecodesign study [9]

\begin{tabular}{llll}
\hline \multicolumn{1}{c}{ compressor type } & \multicolumn{3}{l}{ coefficients } \\
& A & B & C \\
\hline fixed speed rotary compressor & -0.928 & 13.911 & 27.110 \\
variable speed rotary compressor & -1.549 & 21.573 & 0.905 \\
\hline
\end{tabular}

$p_{2}$ : discharge pressure

The isentropic process is an idealized thermodynamic process and assumes no heat exchange with the environment. For the calculation of isentropic efficiency and the relationship with the former metric of the standard, specific energy, see ISO 1217:2009/Amendment 1:2016 [12]. The target efficiency for air compressor packages can be calculated using the following regression curve:

$$
\begin{array}{r}
\eta_{\text {target }}=A \cdot \ln ^{2}\left(q_{V \max }\right)+B \cdot \ln \left(q_{V \max }\right)+C+ \\
\left(100-\left(A \cdot \ln ^{2}\left(q_{V \max }\right)+B \cdot \ln ^{2}\left(q_{V \max }\right)+C\right)\right) \cdot d / 100
\end{array}
$$

Where:

$\eta_{\text {target }}$ : isentropic efficiency that the product shall achieve

$q_{V \max }:$ full-load volume flow [1/s]

$d$ : proportional loss factor

The parameters for A, B and C according to the Ecodesign study [9] are shown in table 1 . The d-value (proportional loss factor) expresses the difference in the average efficiency and the theoretical optimum package efficiency and is specified in the respective regulations.

\section{Availability and safety architectures}

Air compressors are often used in industrial environments. This environment may contain or be surrounded by aggressive gases, high temperatures, moisture, or oil, which constitute very harsh conditions especially for electronics and sensitive measurements. This increases the failure rate of the electronic components as in e.g. microcontrollers, which leads to a reduction of the reliability and availability of the measurement.

One of the most common and successful techniques for increasing the reliability and/or availability of a system is redundancy. In redundancy architecture, multiple subsystems are available to perform the same functionality. Therefore, the system is referred as $\mathrm{M}$ out of $\mathrm{N}(\mathrm{MooN})$, where $\mathrm{N}$ represents the number of subsystems performing or willing to perform the same function and $\mathrm{M}$ represents the actual required output subsystems such as 10o2, 1003, 1004, 2004 etc. If one subsystem fails, a redundant subsystem takes control and performs the system task [15].

There are many methods to provide redundancy, for example, static redundancy uses a voting element to analyze and compare the output of multiple subsystems to select the majority decision from those subsystems. In such a methodology, the configuration of the system does not change after a fault is detected in a subsystem [16]. 
In dynamic redundancy, the system configuration is dynamically changed based on the evaluation of the system's diagnostic element. In hot dynamic redundancy, all subsystems are running and connected to the load. Within a very short time, it is possible to switch from one subsystem to another in order to maintain the safe operation of the system. In dynamic cold or standby redundancy, only one subsystem is running and performs the system tasks while the other subsystems are in standby state. When a malfunction is detected in the running subsystem, one of the standby subsystems is switched on to handle the system functions [17].

In SoC architecture design with redundancy, there are two main strategies to provide the arbitration, i.e., redundancy with hardware comparator and redundancy with software comparator. The redundancy with hardware comparator is the common implemented technique in the available safety microcontroller such as HICore1 [18] and Hercules microcontroller family [19]. Such lockstep approach supports only shutdown in case of system failure, which increases the reliability of the system but negatively affects its availability. To increase the flexibility of the system, redundancy with software comparator is developed [20, 21]. In this paper, a new concept of SoC based on 1002 system architecture with software comparator is presented to increase the availability of the system.

\section{Design of the system}

For the concept of the system design, shown in figure 2, several requirements must be fulfilled. The compressed air system contains one to $\mathrm{N}$ compressors from different types. For each compressor in the system, an efficiency calculation has to be performed. Efficiency is calculated on the intelligent and high available flow efficiency sensor downstream of the compressor. Relevant data for the efficiency calculation is collected up to $20 \mathrm{~m}$ away from the flow sensor. A high-level PLC provides an overview of all collected and calculated data and allows the provision of the data for possible cloud applications.

From the requirements listed before, first points of the specification for the flow sensor are derived. Several sensors must be connected by different interfaces. The integration of the flow efficiency sensor into an over laying network structure will be done by OPC UA as an ethernet based protocol.

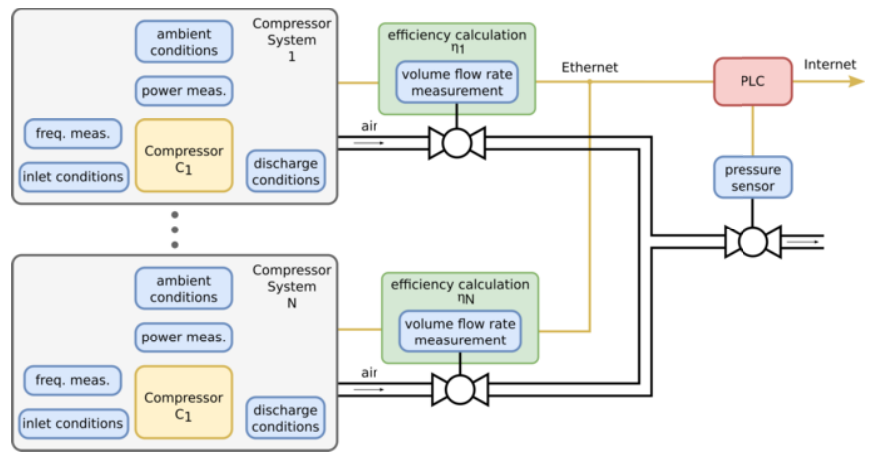

Figure 2. Schematic of the smart air compressed system. 


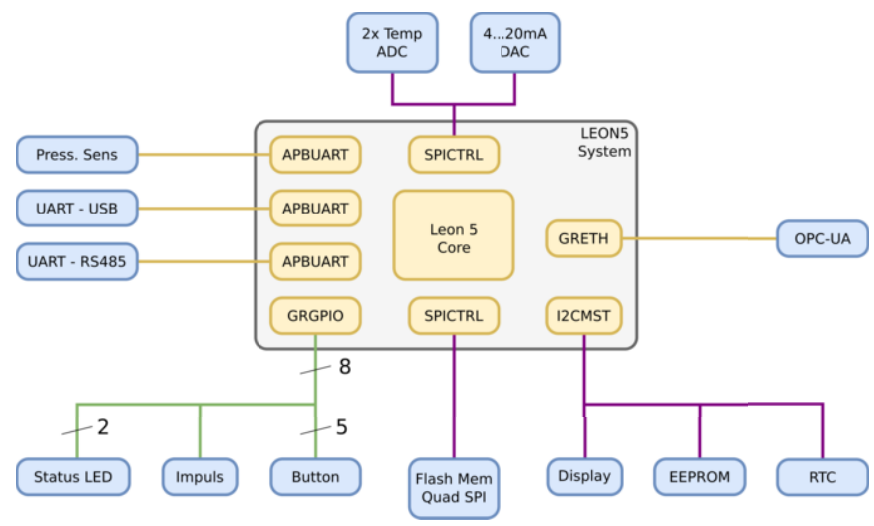

Figure 3. Schematic of the one channel sensor SoC.

\subsection{Efficiency SoC design}

Based on the theory provided in section 3, the hardware concept for the flow sensor will follow a 1002 redundancy approach. For both channels a high level of diagnostic shall be implemented to detect malfunctions in one channel to keep the system running by the second channel. Internally a LEON5 IP core from Cobham Gaisler will be used. It is available under GPL license and as commercial licensed IP for later usage. The benefit seen with this IP is the availability of hundreds of peripherals, also available under GPL license. Since it already brings a small FPU, Buildroot Linux is available for LEON5. According to the specification, the LEON5 core will be extended with different peripherals like UART, SPI, GPIO, ethernet and I2C interfaces. The one channel approach can be seen in figure 3 .

For the specification of the sensor SoC, among the needed data for efficiency calculation according to the mentioned calculation methods, several requirements are derived from the international standard OIML 137 [22, 23].

The two-channel system will follow the concept in figure 4. System A and System $\mathrm{B}$ will be extended with diagnosis units to get a high degree of diagnostics. An arbiter will be used to realize the dynamic hot standby functionality. For the user, the device will behave like a single channel system, with additional health and predictive maintenance information.

The SoC approach will be realized on a FPGA based hardware platform with the possibility to realize it later as a silicon-based product. For the commercial usage an FPGA based product is not feasible. The costs for the FPGA are not competitive as well as that the susceptibility to errors is too high.

\subsection{Efficiency measurement design}

Volume flow measurement downstream of a compressed air compressor is a demanding and complex problem. For a reliable measurement the system must be inert against shock, temperature, aggressive medium conditions (oil) and condensate. To meet these conditions the differential pressure (DP) measurement principle as described in ISO 5167 was selected. 
Table 2. Efficiency measurement design parameters

\begin{tabular}{lll}
\hline Measurand & Unit & Range \\
\hline Pressure & $\mathrm{bar}$ & $1 \ldots .20$ \\
Differential pressure & $\mathrm{mbar}$ & $0 \ldots 100$ \\
Medium temperature & ${ }^{\circ} \mathrm{C}$ & $-30 \ldots 300$ \\
Inlet temperature & ${ }^{\circ} \mathrm{C}$ & $-20 \ldots 65$ \\
Inlet pressure & $\mathrm{mbar}$ & $300 \ldots 1100$ \\
Inlet humidity & $\% \mathrm{RH}$. & $0 \ldots 100$ \\
Compressor power & $\mathrm{kWh}$ & $0 \ldots 350$ \\
\hline
\end{tabular}

For minimal pressure loss and the ability of retrofitting a special averaging pitot tube (APT) was designed to satisfy the above-mentioned conditions (figure 5). DP volume flow measurement according to ISO 5167-1 [24] requires the additional measurement of medium temperature and system pressure. Since the APT was designed to accommodate a temperature as well as a pressure measurement no further installation points, sensors and transmitters are required.

To achieve higher measurement accuracy the APT will be installed in a measuring armature with shut-off function. This ensures controlled installation conditions and easy access for maintenance and replacement.

For DP measurement a custom sensor from KROHNE, a well-known manufacturer of industrial process instrumentation, was selected. The sensor is specially designed for pressure profiles of compressed air compressors with a large DP measuring range as well as a higher sample rate to factor in pulsation effects.

The volume flow measurement device is designed in compliance with OIML 137 $[21,22]$. For quality assurance the device will also be certified according to OIML 137.

Process connections from the DP sensor to the DP device are in compliance with EN 61518 in order to facilitate on-site in operating testing of the DP sensor. This also enables the installation of different DP devices according to ISO 5167 (if required by standards or regulations) since the device is in principle transparent to the SoC.

Further input parameters of the efficiency calculation to be measured according to ISO 1217 are inlet conditions (temperature, pressure, humidity) and the electric power

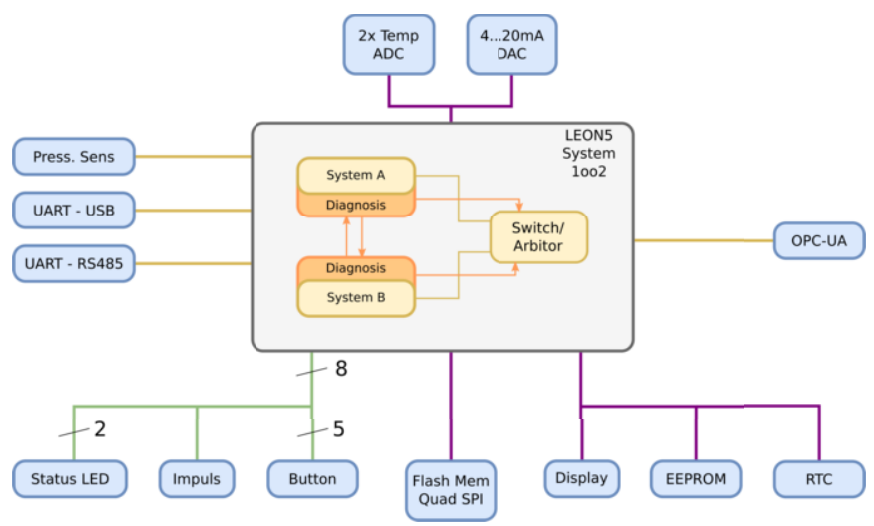

Figure 4. Schematic of the two-channel sensor SoC. 

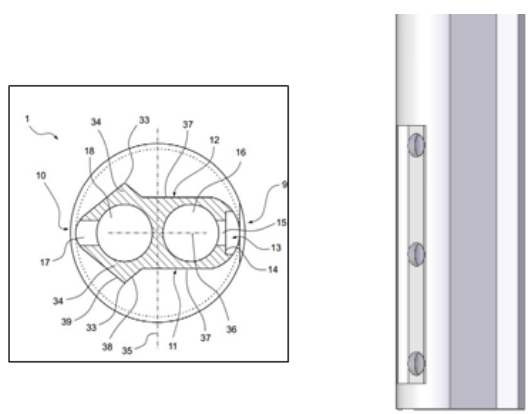

Figure 5. Left: Profile of the APT with differential pressure pipes; Right: View of the APT [German Patent 10 $2021109414.3,14.04 .2021]$

usage of the compressor. An overview of the primary measurands is presented in table 2 . For data collection the SoC will provide a Modbus interface and analog input options.

Efficiency assessment, control and maintenance information as well as assessment according to international regulations will be calculated in real-time on the SoC and will be available via display and ethernet interface (OPC UA).

\section{Conclusion}

In this work, a new concept for an intelligent efficiency measurement system for industrial compressed air systems was presented. The specification of the concept was defined based on the international standards ISO 1217:2009/Amd 1:2016 and OIML 137. A new SoC design with high availability based on the 1002 architecture was introduced. Industry 4.0 connectivity is supported in this design by the OPC UA protocol.

For future developments comprehensive compressor control strategies and predictive maintenance assessment will be provided on the PLC for one or several compressed air compressors based on efficiency measurements from the proposed SoC. This concept will be further refined with machine learning capabilities of the PLC.

Since $80-93 \%$ of the electric energy used by an air compressor is converted into heat, a properly designed heat recovery has great savings potential [8]. For a more holistic efficiency calculation measurements with a heat meter are planned.

\section{Acknowledgment}

We acknowledge the Distr@1 funding program of the Hessian State Chancellery in the area of the Minister for Digital Strategy and Development, project 20_0097_2A Zuverlässige SoC-Sensorik zur Effizienzsteigerung bei Hochleistungskompressorsystemen auf KI-Basis - Scientia.

We acknowledge our colleagues and co-workers at the Department of Computer Architecture and System Programming, University of Kassel, Postberg+Co und inTec automation for their support, comments, and discussion. 


\section{References}

[1] Sullair, Uses of Compressed Air - Sullair. [Online]. Available: https://america.sullair.com/en/blog/uses-compressed-air (accessed: Aug. 10 2021).

[2] Air Compressor Market Size, Share \& Trends - Industry Report, 2025. [Online]. Available: https://www.grandviewresearch.com/industry-analysis/air-compressor-market (accessed: Aug. 10 2021).

[3] "Compressed air in industry: how can digitalization help energy optimization?," METRON, 24 Jun., 2021. https://www.metron.energy/blog/compressed-air-industry-energy-optimization/ (accessed: Aug. 10 2021).

[4] M. P. Roessler, "Energy efficiency in industrial compressed air generation-Survey about developments, trends and alternative generation technologies," 2016.

[5] Manuel Unger and Peter Radgen, Eds., Energy efficiency in compressed air systems-a review of energy efficiency potentials, technological development, energy policy actions and future importance, 2017.

[6] Federal Ministry for Economics Affairs and Energy, Industrie 4.0. [Online]. Available: https://www.bmwi.de/Redaktion/EN/Dossier/industrie-40.html (accessed: Aug. 10 2021).

[7] D. Cogliati, M. Falchetto, D. Pau, M. Roveri, and G. Viscardi, "Intelligent Cyber-Physical Systems for Industry 4.0," in 2018 First International Conference on Artificial Intelligence for Industries (AI4I), Laguna Hills, CA, USA, Sep. 2018 - Sep. 2018, pp. 19-22.

[8] Ying Zheng and Michael Bellstedt, "Compressor Degradation Assessment and Wear Mitigation Strategy,” Meat \& Lifestock Australia Limited.

[9] M. van Elburg, M. van der Voort, R. van den Boorn, R. Kemna, W. Li, and Van Holsteijn en Kemna, BV, "Study on Amended Working Plan under the Ecodesign Directive," Final Report of Task, vol. 3, 2011.

[10] Energy Conservation Program: Energy Conservation Standards for Air Compressors, Department of Energy.

[11] Minimum allowable values of energy efficiency and energy efficiency grades for displacement air compressors, GB 19153-2019, SAMR AND SAC, Dec. 2019.

[12] Displacement compressors — Acceptance tests, ISO 1217:2009/Amd 1:2016, International Organization for Standardization (ISO), Apr. 2016.

[13] Turbocompressors - Performance test code, ISO 5389:2005, International Organization for Standardization (ISO), Dec. 2005.

[14] American Society of Mechanical Engineers, Performance test code on compressors and exhausters: An American national standard. New York: The American Society of Mechanical Engineers, 1998.

[15] J. Börscök, "Functional Safety: Basic Principles of Safety-relaged Systems," Hüthig, Heidelberg, 2007.

[16] R. W. Butler and S. C. Johnson, "Techniques for modeling the reliability of fault-tolerant systems with the Markov state-space approach,” 1995.

[17] L. Xing, G. Levitin, and C. Wang, Dynamic System Reliability: Modeling and Analysis of Dynamic and Dependent Behaviors: Wiley, 2019. [Online]. Available: https://books.google.com.eg/books?id=eeBDwAAQBAJ

[18] A. Hayek, B. Machmur, M. Schreiber, J. Borcsok, S. Golz, and M. Epp, "HICore1: "Safety on a chip" turnkey solution for industrial control," in 2014 IEEE 25th International Conference on ApplicationSpecific Systems, Architectures and Processors, Zurich, Switzerland, Jun. 2014 - Jun. 2014, pp. 74-75.

[19] Texas Instruments, Safety Manual for TMS570LS31x and TMS570LS21x Hercules ${ }^{\mathrm{TM}}$ and ARM® Safety Critical Microcontrollers: User's Guide.

[20] M. Abdelawwad, M. Drabesch, M. Schwarz, M. I. Hafiz, and J. Börcsök, "Communication SoC based on 10o2D architecture for industrial human-robot-collaboration," in 2021 18th International MultiConference on Systems, Signals \& Devices (SSD), 2021, pp. 954-959.

[21] J. Börcsök, W. Müller, E. Hahn, M. Schwarz, and M. Abdelawwad, "Safe-System-on-Chip for Functional Safety," in 2021 18th International Multi-Conference on Systems, Signals \& Devices (SSD), 2021, pp. 619-624.

[22] Gas meters - Part 1: Metrological and technical requirements, OIML R 137-1, International Organization of Legal Metrology, 2012.

[23] Gas meters - Part 2: Metrological controls and performance tests, OIML R 137-2, International Organization of Legal Metrology, 2012.

[24] Measurement of fluid flow by means of pressure differential devices inserted in circular cross-section conduits running full: Part 1: General principles and requirements, ISO 5167-1:2003, Mar. 2003. 